\title{
Narrative Changes Predict a Decrease in Symptoms in CBT for Depression: An Exploratory Study
}

\author{
Miguel M. Gonçalves, ${ }^{1 *}$ Joana Ribeiro Silva, ${ }^{1}$ Inês Mendes, ${ }^{1}$ Catarina Rosa, ${ }^{1}$ \\ António P. Ribeiro, ${ }^{1}$ João Batista, ${ }^{1}$ Inês Sousa ${ }^{3}$ and Carlos F. Fernandes ${ }^{2}$ \\ ${ }^{1}$ University of Minho, School of Psychology, Braga, Portugal \\ ${ }^{2}$ University of Aveiro, Departament of Education, Aveiro, Portugal \\ ${ }^{3}$ University of Minho, Department of Mathematics, Braga, Portugal
}

\begin{abstract}
Objective: Innovative moments (IMs) are new and more adjusted ways of thinking, acting, feeling and relating that emerge during psychotherapy. Previous research on IMs has provided sustainable evidence that IMs differentiate recovered from unchanged psychotherapy cases. However, studies with cognitive behavioural therapy (CBT) are so far absent. The present study tests whether IMs can be reliably identified in CBT and examines if IMs and symptoms' improvement are associated.

Methods: The following variables were assessed in each session from a sample of six cases of CBT for depression (a total of 111 sessions): (a) symptomatology outcomes (Outcome Questionnaire-OQ-10) and (b) IMs. Two hierarchical linear models were used: one to test whether IMs predicted a symptom decrease in the next session and a second one to test whether symptoms in one session predicted the emergence of IMs in the next session.

Results: Innovative moments were better predictors of symptom decrease than the reverse. A higher proportion of a specific type of IMs-reflection 2-in one session predicted a decrease in symptoms in the next session. Thus, when clients further elaborated this type of IM (in which clients describe positive contrasts or elaborate on changes processes), a reduction in symptoms was observed in the next session.
\end{abstract}

Discussion: A higher expression and elaboration of reflection 2 IMs appear to have a facilitative function in the reduction of depressive symptoms in this sample of CBT. Copyright $@ 2016$ John Wiley \& Sons, Ltd.

Key Practitioner Message

- Elaborating innovative moments (IMs) that are new ways of thinking, feeling, behaving and relating, in the therapeutic dialogue, may facilitate change.

- IMs that are more predictive of amelioration of symptoms in CBT are the ones focused on contrasts between former problematic patterns and new adjusted ones; and the ones in which the clients elaborate on processes of change.

- Therapists may integrate these kinds of questions (centred on contrasts and centred on what allowed change from the client's perspective), in the usual CBT techniques.

- When elaborating these IMs successfully, therapists may expect an improvement in symptoms in the next session of psychotherapy. Copyright (C) 2016 John Wiley \& Sons, Ltd.

Keywords: Innovative Moments, Cognitive Behavioural Therapy, Process of Change, Psychotherapy, Depression

In the last decade, we have been developing a research programme that explores change in psychotherapy using the conceptual framework of innovative moments (IMs; Gonçalves, Ribeiro, Mendes, Matos, \& Santos, 2011). IMs are all the instances in the therapeutic dialogue that challenge the maladaptive framework of

*Correspondence to: Miguel M. Gonçalves, Psychotherapy and Psychology Research Center (CIPsi), School of Psychology, University of Minho, Braga 4710-057, Portugal.

E-mail: mgoncalves@psi.uminho.pt meaning that brought the client to therapy. This maladaptive framework may be conceived as an implicit rule of meaning (e.g., 'always privilege significant others' wishes, and neglect my own'), with wide impact on behaviour, cognition, affect and so on, while IMs are all the moments in which this framework is challenged (e.g., 'yesterday I did what I thought was good for me and it felt good').

Moreover, despite the narrative background of this concept (see White \& Epston, 1990 on the concept of unique outcome), we assume that IMs are a transtheoretical 
concept, occurring in all 'talking therapies'. For example, if cognitive therapists challenge the catastrophic meaning of cognitions, then alternative interpretations could emerge, potentially resulting in IMs. Furthermore, emotion-focused therapists use different techniques (e.g., empty chair dialogue) to transform maladaptive emotional processes. During these processes, IMs could certainly emerge when the client accesses and explores the unmet need. The same reasoning applies to psychodynamic therapy because therapist interpretations may lead to new self-understandings and insights. If change is ubiquitous in psychotherapy (at least in successful cases), then IMs would emerge very frequently and lead to further changes. In fact, we would expect that even in 'action therapies' (e.g., behavioural activation; Kanter, Busch, \& Rusch, 2009) IMs would be important, as people attribute meaning to their actions and this is actually congruent with what is proposed by these models (e.g., importance of values and meaning of life).

Based on these assumptions, a research programme to track IMs has been developed by Gonçalves and colleagues (Alves et al., 2014; Gonçalves, Matos, \& Santos, 2009; Gonçalves, Mendes, Ribeiro, Angus, \& Greenberg, 2010; Matos, Santos, Gonçalves, \& Martins, 2009; Mendes et al., 2011). The innovative moments coding system (IMCS; Gonçalves et al., 2011) was applied to multiple samples characterized by distinct clinical problems and therapeutic models. However, most of these models shared a predominantly constructivist or phenomenological approach such as narrative therapy (NT; Gonçalves, Ribeiro, Silva, Mendes, \& Sousa, 2016; Matos et al., 2009), meaning reconstruction approach to grief (Alves, Mendes, Gonçalves, \& Neymeier, 2012; Alves et al., 2014), client-centred therapy (CCT; Gonçalves et al., 2012) or emotion-focused therapy (EFT; Mendes, Ribeiro, Angus, Greenberg, \& Gonçalves, 2010). These previous studies consistently showed not only the reliability of the IMCS in identifying IMs as markers of change throughout treatment but also the occurrence of a higher proportion of IMs in successful cases.

Related findings were obtained in a recent study on narrative processes in psychotherapy (Boritz, Bryntwick, Angus, Greenberg, \& Constantino, 2013) that used a new narrative coding system-Narrative-Emotion Process Coding System (Boritz et al., 2013) — to analyse narrative and emotional processes in three different therapeutic approaches (EFT, CCT and CBT). The results revealed a significantly higher proportion of narrative-emotion process change markers in recovered than in unchanged cases. Consistent with the research findings on IMs, the higher incidence of 'discovery storytelling markers' in recovered cases (a concept that is very similar to IMs) confirmed the higher emergence of exceptions or novelties in successful treatment regardless of the psychotherapy model used.

Recently, a study conducted with a sample of NT for depression reinforced the importance of IMs in the therapeutic change. While former studies were correlational, comparing the emergence of IMs in recovered and unchanged cases, Gonçalves et al. (2016) investigated the predictive influence of IMs on symptoms change. In this study, two different sets of models were tested. One tested the occurrence of IMs in one session as a predictor of symptom improvement in the next session, and the second reversed the prediction direction and tested the symptom improvement in one session as a predictor of IMs in the next session. The results clearly suggested that the first model explained significantly more variance than the second one; thus, IMs were better predictors of symptom improvement than vice-versa. These findings support the conceptualization of IMs as representing transformation processes that precede and facilitate symptomatic change. Despite the exploratory nature of this study (a small sample of NT for depression), its results are relevant for two reasons. First, the majority of process-outcome studies available are correlational and do not analyse the precedence of process variables (Crits-Christoph, Gibbons, \& Mukherjee, 2013), and second, none of the previous research conducted with IMs established the precedence of IMs over symptoms change. The precedence of IMs occurrence over symptom improvement supported the assumption that IMs may be a process of change that not only correlate but may also foster symptoms amelioration. Of course, as this study uses a sample of NT, it can be argued that the precedence of IMs over symptom's change is just supporting the proposal of narrative therapists that the identification and elaboration of unique outcomes (i.e., IMs) are an active ingredient of therapy. Thus, it is relevant to replicate this study with different theoretical approaches.

Innovative moments may emerge in seven different types (Table 1): (1) Action 1 IMs, which are clients' behaviours that are inconsistent with the problem (for simplicity, we use maladaptive framework and problem interchangeably); (2) Reflection 1 IMs include new forms of understanding the problem; (3) Protest 1 IMs entail refusing the problem and its assumptions in the form of actions or thoughts; (4) Reflection 2 IMs involve thoughts that express a contrast between a past problematic selfposition and a more adjusted current one, or thoughts that describe a process of change into a more adjusted state; (5) Protest 2 IMs refer to positions of assertiveness and empowerment; (6) Reconceptualization IMs involve both a contrast between a past problematic self-position and a more adjusted current one, as well as a description of the change process that enabled the transformation involved in that contrast; and (7) Action 2 IMs (previously termed Performing Change) consist of performed or projected actions that illustrate how the achieved changes can be integrated into the future and to other life dimensions (e.g., from work to family).

Previous research has suggested a differential effect of the seven different types of IMs in the construction of 
Table 1. Innovative moments with examples

\begin{tabular}{lcc}
\hline Types of IM $\quad$ Subtypes & Definition & $\begin{array}{c}\text { Examples } \\
\text { (Maladaptive framework of }\end{array}$ \\
meaning:
\end{tabular}

meaning: depression)

Low-level IMs

(Creating

distance from

the problem)

Reflection 1
Performed and intended actions to overcome the problem

New understanding of the problem

Protest 1

Action 2

High-level IMs

(Centred on

change)

Objecting to the problem and its assumptions

Generalization of good outcomes into the future (previously termed

performing change) and to other life dimensions (performed or projected actions)

Reflection 2

Protest 2

Re-
conceptualization
Contrasting Self (what changed?)

OR

Self-Transformation process (how / why change occurred) Assertiveness and empowerment

Moments distanced from the experience (metapositions) where the self is repositioned outside the problematic experience AND also understands the processes involved in this transformation
C: Yesterday, I went to the cinema for the first time in months!

C: I realize that what I was doing was just not humanly possible because I was pushing myself and I never allowed myself any free time, uh, to myself ... and it's more natural and more healthy to let some of these extra activities go...

$\mathrm{C}$ : What am I becoming after all? Is this where I'll be getting to? Am I going to stagnate here!?

$\mathrm{T}$ : You seem to have so many projects for the future now!

C: Yes, you're right. I want to do all of the things that were impossible for me to do while I was dominated by depression. I want to work again and to have the time to enjoy my life with my children. I want to have friends again. The loss of all of the friendships of the past is something that still hurts me really deeply.

C: I feel positive and strong. It's okay to ask for these things [her needs], it's a new part of me, so I'm not going to turn it down.

C: I am an adult and I am responsible for my life, and, and, I want to acknowledge these feelings and I'm going to let them out! I want to experience life, I want to grow and it feels good to be in charge of my own life.

C: I currently feel differently. I don't worry about what others think about what I'm saying. I discovered that I need to respect my needs and opinions, even if other people disagree with me. Before to protect me from disagreeing with others I was always in conflict with myself-thinking one thing, saying another. How is the disagreement with others worse than this internal fighting? change throughout treatment. Action 1 IMs have consistently emerged in lower proportions throughout the therapeutic process (Alves et al., 2014; Gonçalves et al., 2010; Mendes et al., 2010). Reflection IMs have proved to be extremely frequent across treatment, but only type 2 appears to be consistently associated with successful cases (Alves et al., 2014; Mendes et al., 2011). Protest IMs proportion has proved to be highly variable, depending on the therapeutic model and on the problems clients brought to therapy (e.g., major depression versus complicated grief; Alves et al., 2014). For instance, in EFT, protest IMs emerged primarily during chair exercises and presented a higher proportion than in any other therapeutic model studied so far (Mendes et al., 2011). Nevertheless, and similarly to what was observed for reflection IMs, only protest 2 IMs were associated with successful treatment (Mendes et al., 2011). Reconceptualization is seemingly the most important IM type in the construction of change (Gonçalves \& Ribeiro, 2012). Empirical data have supported this claim as reconceptualization consistently occurred more frequently in recovered cases, particularly from the middle of therapy onwards (Alves et al., 2014; Gonçalves et al., 2016; Matos et al., 2009; Mendes et al., 2010). Finally, action 2 IMs usually emerge towards the final phase of therapy as 
a way of generalizing change into the future (Alves et al., 2014; Gonçalves et al., 2016; Matos et al., 2009; Mendes et al., 2010). Therefore, action 1 , reflection 1 and protest 1 IMs may constitute important markers that something new is occurring and change is taking place. Notwithstanding, these types of IMs might be insufficient for a significant change in personal meanings leading to the transformation of the previous maladaptive framework. Empirical data supported the idea that this change may only be achieved through the emergence of reflection 2, protest 2, reconceptualization and action 2 IMs. We have termed the first group of IMs low-level IMs (action 1, reflection 1 and protest 1 ) and the second group (action 2, reflection 2, protest 2 and reconceptualization) high-level IMs to highlight their developmental differences.

The present study is the first to apply the IMCS (Gonçalves et al., 2011) to CBT. The model of CBT analysed here was in line with the tradition of Beck, Rush, Shaw, and Emery (1979), valuing behavioural change, as well as Socratic dialogues that allow cognitions and assumptions to be empirically tested and changed. Moreover, as stated before, to the best of our knowledge, there is only one study that shows the precedence of IMs over symptom improvement, using an NT sample (Gonçalves et al., 2016). The replication of the same results with another model of therapy would be relevant, as therapists foreign to NT do not pay attention to IMs as part of their technical knowledge.

This exploratory study will investigate three questions:

(1) Can IMs be reliably identified in CBT, as occurred with other therapeutic modalities?

(2) Is the occurrence of IMs a better predictor of symptom improvement in the next session $(\mathrm{lag}+1)$ than the reverse, as occurred with NT (Gonçalves et al., 2016)?

(3) More specifically, which type or types of IMs are better predictors of symptom improvement in the next session $(\operatorname{lag}+1)$ ?

We hypothesize that (1) IMs will be reliably identified in CBT; (2) IMs will be better predictors of symptom improvement, than the reverse, congruently with what occurred in the previous study of Gonçalves et al. (2016); and (3) high-level IMs, but not low-level IMs, would predict symptom improvement (we do not offer any prediction on the specific types of IMs, as this is an exploratory study).

\section{METHOD}

\section{Clients}

The present sample is constituted by six clients from a sample of 29 CBT clients who had participated in a previous study comparing the effectiveness of NT with CBT for depression (Lopes, Gonçalves, Fassnach, Machado, \& Sousa, 2015; Lopes et al., 2014). Their inclusion into the broader study was defined according to the following criteria: (a) a diagnosis of major depressive disorder according to the Diagnostic and Statistical Manual of Mental Disorders (DSM-IV; American Psychiatric Association, 2000) and (b) a signed informed consent for participating in the study, which included completing questionnaires and having the session be videotaped. At the intake, all clients undertook a Structural Clinical Interview for the DSM-IV axis I (First, Spitzer, Gibbons, \& Williams, 2002) and axis II if needed (First, Gibbon, Spitzer, Williams, \& Benjamin, 1997; see Lopes et al., 2014 for further details). The following exclusion criteria were used: (a) any axis II diagnosis; (b) any other concurrent axis I disorder that could be the focus of clinical attention (e.g., substance-related disorders, sexual disorders or eating disorders); (c) severe suicidal thoughts; (d) psychotic symptoms; or (e) bipolar disorder. Clients exhibiting anxiety complaints or a secondary anxiety disorder were included in the study if the anxiety was not considered a primary complaint (e.g., panic disorder). The Psychology Research Center at the University of Minho, the institution that supported this study, considered that all the ethical guidelines for implementing the controlled trial were met (at that time, the university did not have a formal ethical committee for the Human Sciences). Therapy consisted of a maximum of 20 individual psychotherapy sessions provided at the university clinic. Therapy was delivered free of charge, on a weekly basis until session 16, after which the interval increased to 2 weeks until the maximum of 20 sessions was reached (plus follow-up at 1, 3, 6, 12, 21 and 31 months). When clients needed to receive more sessions, they were referred to other therapists at the Clinic or to alternative mental health services.

In the parent study, the sample of CBT clients $(N=29)$ consisted of nine dropouts (31\%) and 20 completers $(69 \%)$. Of the 20 completers, three unchanged and three recovered cases were randomly selected for the current study. Only six cases were drawn from the parent study's sample $(N=29)$ because of the time-consuming nature of the IMs' coding procedure (the coding of each complete case takes approximately 1 month of full-time daily work by two coders). Although we recognize that the sample size is far from ideal, in the analyses presented below, the effect of the sample size is considered in the $p$-values. Therefore, the statistical power of the models presented is enough, so that the magnitude of the effect of interest can be detected with this sample size.

The status of cases (i.e., recovered versus unchanged) was determined using the two conditions defined by Jacobson and Truax (1991). For a case to be considered recovered, the following criteria must be met: (a) the client's movement from a dysfunctional to functional range in a 
given outcome measure; and (b) the reliability of change, i.e., change higher than the reliable change index (RCI) for the respective measure. Recovered cases met both conditions, while unchanged cases met none. Both the Beck Depression Inventory II (BDI-II; Beck, Steer, \& Brown, 1996) and the Outcome Questionnaire (OQ-45.2; Lambert et al., 1996) were used to establish the status of the cases (Table 2).

The recovered and unchanged cases exhibited no significant differences in terms of the severity of symptoms at the initial stage of therapy based on the BDI-II $(t(4)$ $=-0.45, p=0.68)$ and the OQ-45.2 scores $(t(4)=-0.07$, $p=0.95)$. Additionally, no differences between cases were found regarding the number of therapy sessions received throughout the treatment $(t(4)=1.00, p=0.37)$. The clients completed an average of $18.67(S D=3.27)$ sessions.

From the six clients in this sample, five were women $(83.3 \%)$ and one was a man $(16.7 \%)$, with ages ranging from 22 to 46 years $(M=34.50, S D=8.48)$. Two clients were single $(33.3 \%)$, three were married $(50 \%)$ and one was divorced $(16.7 \%)$. All of the clients had completed at least the 9th grade; however, some clients had up to 16 years of academic education $(M=14.17, S D=2.71)$. Regarding their professional status, one client was unemployed $(16.7 \%)$, three were professionally active $(50 \%)$ and two were students $(33.3 \%)$. Their socio-economic level, assessed by the Graffar Index (Graffar, 1956), ranged from 9 (Level I, high scale) to 14 (Level III, medium scale). These demographic characteristics did not differ between the groups.

\section{Therapist and Therapy}

All six clients were treated by the same therapist, a 33year-old Caucasian man who was a PhD student in clinical psychology with 3 years of experience as a cognitive behavioural psychotherapist. The treatments were individual and followed a cognitive behavioural manual (Beck et al., 1979; Leahy \& Holland, 2000). During sample collection, the therapist was practicing as a full-time therapist, involved only in psychotherapy and supervision activities.

Therapeutic work was based on behavioural strategies (e.g., a diary of activities) and cognitive restructuring techniques (e.g., identification and debate of automatic thoughts; identification, questioning and clarification of dysfunctional beliefs). Clients were encouraged to selfmonitor their progress outside the sessions through homework assignments later reviewed in the sessions. The evaluation of the client's progress and the prevention of relapse were the target of the final phase of the treatment. In all of the cases, therapy was ended by mutual consensus, when the clients and the therapist agreed that the main goals had been achieved, or when the manual was completed.

A senior CBT therapist monitored all of the cases through weekly supervision sessions to ensure therapist's adherence to the CBT model of intervention. An Adherence and Competence Scale for Narrative and CognitiveBehavioural Therapy (ACS-N-CBT; Gonçalves, Bento, Lopes, \& Salgado, 2009) was used by independent coders to further confirmed adherence and competent delivery of treatment. Both proved to be adequate (for further details, see Lopes et al., 2014).

\section{Measures}

\section{Pre-post Measures}

Beck Depression Inventory II (Beck et al., 1996). Symptoms of depression were evaluated with the BDI-II, a 21-item selfreport instrument (Beck et al., 1996; Steer, Brown, Beck, \& Sanderson, 2001) that uses a 4-point Likert scale. Items are rated from 0 to 3 , and total scores range from 0 to 63 . The internal consistency (Cronbach's $\alpha=0.91$; Steer et al., 2001; Cronbach's $\alpha=0.89$; Lopes et al., 2014) and construct validity (Beck et al., 1996; Steer et al., 2001) have proved to be adequate for this measure. The Portuguese version of the BDI-II has been reported as exhibiting equivalent characteristics to the original American version (Campos \& Gonçalves, 2011; Coelho, Martins, \& Barros, 2002). Because the RCI (Jacobson \& Truax, 1991) could not be found across Portuguese studies, normative data gathered from the meta-analyses of diverse samples (Seggar, Lambert, \& Hansen, 2002) were used to calculate the proportion of clinical change $(\mathrm{RCI}=8.46$; cut-off score $=14.29)$. In this sample, considering the intent-to-treat sample $(N=63)$ the Cronbach's alpha was 0.89 (Lopes et al., 2014).

Outcome Questionnaire 45.2 (Lambert et al., 1996). Clients' progress and clinical conditions were evaluated with the OQ-45.2, a self-report questionnaire with 45 questions concerning not only psychological distress but also interpersonal relations and the social role of the clients. This measure's internal consistency has been found to be excellent for Portuguese (Cronbach's $\alpha=0.89$; Machado \& Fassnacht, 2014) and non-Portuguese samples (de Jong et al., 2007; Lambert et al., 1996). The RCI calculated for the Portuguese population was 15 points, and the cutoff was 62 points (Machado \& Fassnacht, 2014). In this sample, considering the intent-to-treat sample $(N=63)$ the $\alpha$ was 0.89 (Lopes et al., 2014).

\section{Session Measures}

Outcome questionnaire 10.2 (Lambert, Finch, Okiishi, E Burlingame, 2005). The OQ-10.2 is as a brief version of the OQ-45.2, consisting of 10 items that monitor changes in the clients' symptomatic distress over short periods 
(Lambert et al., 1996). Empirical data have shown adequate values of internal consistency (Cronbach's $\alpha=0.87$; Goates-Jones \& Hill, 2008) and test-retest reliability (Pearson $r=0.62$; Lambert et al., 2005).

Innovative Moments Coding System (IMCS; Gonçalves et al., 2011). The IMCS identifies the seven types of IMs-action 1 and 2, reflection 1 and 2, protest 1 and 2 and reconceptualization (for additional descriptions and illustrative clinical vignettes of each type, see Table 1). In previous quantitative studies, the IMCS (Gonçalves et al., 2011) proved to be reliable, with Cohen's $k$ values of 0.89 in a study on NT, 0.86 in a study on EFT, and 0.97 in a study on CCT (Gonçalves et al., 2012; Gonçalves et al., 2016; Matos et al., 2009; Mendes et al., 2010).

\section{Procedures}

Outcome measures. The outcome measures were administered at the beginning of the therapeutic sessions. The BDI-II and the OQ-45.2 were administered at the first session and then at each fourth session. The OQ.10.2 was used in the remaining sessions. In the sessions in which the OQ-45.2 was used (1st, 4th, 8th, 12th and 16th), the items relative to the OQ-10.2 were extracted for purposes of comparability with the other sessions in which only the OQ-10.2 was used.

Innovative moments coding and reliability. The therapeutic sessions of the six cases were transcribed and subsequently analysed according to the IMCS (Gonçalves et al., 2011). Two independent coders (PhD students), unaware of the outcome status of the cases and the hypotheses of the study, analysed all of the transcripts intensively ( $N=111$ sessions). The coders were trained by the authors of the manual and completed a predefined training protocol (involving several weeks of intensive work) consisting of (a) a coding manual with a set of randomly selected excerpts from the dialogues of therapeutic sessions and (b) a full therapeutic case with a series of different coding steps of increasing difficulty. At the end of the training period, the reliability of the coders was

Table 2. Pre-post outcome measures

\begin{tabular}{|c|c|c|c|c|c|}
\hline \multirow[b]{2}{*}{ Case } & \multicolumn{2}{|c|}{ BDI II } & \multicolumn{2}{|c|}{ OQ-45.2 } & \\
\hline & Pre & Post & Pre & Post & \\
\hline 1 & 34 & 7 & 95 & 11 & Recovered \\
\hline 2 & 26 & 18 & 93 & 70 & Unchanged \\
\hline 3 & 26 & 1 & 75 & 19 & Recovered \\
\hline 4 & 38 & 5 & 109 & 51 & Recovered \\
\hline 5 & 44 & 34 & 110 & 95 & Unchanged \\
\hline 6 & 16 & 24 & 73 & 94 & Unchanged \\
\hline
\end{tabular}

Copyright (C) 2016 John Wiley \& Sons, Ltd. evaluated. The coders were considered to be reliable and able to engage in coding if they achieved Cohen's $k$ values above 0.75 . The IMs coding process entailed three main steps: (1) a consensual definition by the two coders of the clients' maladaptive framework (operationalized as a list of problems based on the client and therapist's conversation); (2) identification of each IM, which required the definition of its beginning and end; and (3) categorization of the identified IMs in terms of type. Coders' reliability was calculated based on the full coding material. Disagreements emerging from the coding process were resolved through consensus, achieved during regular discussion meetings. This approach has been described as interactive and collaborative because it allows the coders to further integrate each other's strengths in subsequent coding, which is thought to exert a positive impact on the coding process (cf. Brinegar, Salvi, Stiles, \& Greenberg, 2006). The IMCS enables identification of the type of IM (e.g., action 1 and reflection 1) and its proportion-a measure of the IMs' length in the transcript. The proportion of each type of IM (for every session of every case) was calculated as the percentage of words involved in that particular IM relative to the total number of words per session. The proportion of IMs has also been the most frequently used measure in previous studies (Gonçalves et al., 2012; Gonçalves et al., 2016; Matos et al., 2009; Mendes et al., 2010), given that the length of a type of IM can be considered a better indicator of its elaboration (taken as a proxy of significance) than the frequency of this type of IM's occurrence.

Hierarchical linear modelling analyses. Data analysis was performed using hierarchical linear modelling (HLM) to determine whether there were any associations between the longitudinal fluctuations of the proportion of IMs and clinical symptomatology, while accounting for the non-independence of data. Analyses were performed using the nonlinear mixed-effects modelling (nlme) package for $\mathrm{R}$ (version 3.1.2, $\mathrm{R}$ Development Core Team, 2013). HLM has numerous advantages over other multivariate repeated measures methods, particularly in the analysis of nested or hierarchically structured data (i.e., observations across time nested within clients; Osborne, 2000). HLM allows for the simultaneous estimation of within — and between — client effects; in the present study, this analysis was fitted to the data, developing a regression model for the fixed effects (predictors) and clientspecific random effects. Thus, we were able to attribute a portion of the variability to differences between participants. We have previously used HLM (Gonçalves et al., 2016) to answer similar questions in a study with NT. At level 1 (within-client), we considered outcomes to be a function of time; at level 2 (between-clients), variability in the level 1 coefficient was modelled as a function of client-level time-invariant covariates. 
For investigation of the main questions of the present study (whether IMs predicted symptoms reduction or whether symptoms reduction predicted IMs), two distinct sets of models were estimated. One model was designed to answer the question: Were IMs in a given session predictors of the symptoms (OQ-10.2) in the next session $(\operatorname{lag}+1)$ ? The other model aimed to answer the reverse question: Did symptoms (OQ-10.2) in a given session predict IMs in the next session $(\mathrm{lag}+1)$ ? These two sets of models were only performed for the most prevalent IMs in these CBT cases to avoid exhaustive presentation of data and to reduce type I error.

\section{RESULTS}

\section{Were Innovative Moments Reliably Identified in Cognitive Behavioural Therapy, as Occurred with Other Therapeutic Modalities?}

Agreement in the identification and delimitation of IMs' proportion was calculated as the number of overlapping words identified by each coder for each IM divided by the total number of words. Agreement was achieved in $90 \%$ of the excerpts for the entire sample, indicating that the coders had $90 \%$ overlapping text in the identification of IMs. After this calculation, Cohen's $k$ was computed to evaluate the reliability in distinguishing between IM types and yielded a result of 0.94 , indicating a high agreement (Hill \& Lambert, 2004).

\section{Were Innovative Moments Better Predictors of Symptoms Change at Lag +1 Than the Reverse?}

Instead of performing HLM analysis for each type of IM, as this is the first study of IMs in CBT, we first analysed the proportion of all types of IMs in the three recovered and the three unchanged cases. As can be seen in Table 3, reflection 1 and reflection 2 IMs were the IMs that presented a higher proportion for all of the cases. Reflection 1 IMs was the type with the highest proportion in

Table 3. Proportion of innovative moments

\begin{tabular}{lccc}
\hline Types of IMs & $\begin{array}{c}\text { Recovered } \\
\text { cases (\%) }\end{array}$ & $\begin{array}{c}\text { Unchanged } \\
\text { cases (\%) }\end{array}$ & All cases (\%) \\
\hline Total IMs & 24.14 & 15.51 & 19.82 \\
Action 1 & 0.58 & 0.82 & 0.70 \\
Action 2 & 1.12 & 0.17 & 0.65 \\
Reflection 1 & 7.85 & 6.89 & 7.37 \\
Reflection 2 & 9.09 & 4.83 & 6.96 \\
Protest 1 & 1.29 & 1.07 & 1.18 \\
Protest 2 & 1 & 0.88 & 0.94 \\
Reconceptualization & 3.22 & 0.83 & 2.03 \\
\hline
\end{tabular}

unchanged cases, while reflection 2 IMs was the highest in recovered cases. The other types of IMs exhibited lower proportions $(\leq 3.22 \%)$. Accordingly, the two HLM models were only performed for reflection 1 and reflection 2 IMs. The first models tested IMs as predictors of symptoms change, while the second tested the reversed order, i.e., symptoms as predictors of IMs.

Reflection IMs as predictors of symptom improvement (first set of models). In the first set of HLM analyses (Table 4), reflection 2 IMs were a significant predictor of clients' symptomatology (OQ-10.2) in the next session $\left(p<0.001 ; R_{a d j}^{2}=0.54\right)$, whereas reflection 1 IMs did not predict clients' symptomatology (OQ-10.2) in the next session $\left(p=0.98 ; R_{a d j}^{2}=0.49\right)$. Thus, the proportion of reflection 2 IMs in one session was negatively associated with clients' symptoms (OQ-10.2) in the next session, meaning that a higher proportion of reflection $2 \mathrm{IMs}$ in one session was associated with an improvement in symptoms in the next session.

Symptomatology as a predictor of reflection IMs (second set of models). In this set of HLM analyses (Table 5), the symptoms (OQ-10.2) presented a significant negative association with reflection 2 IMs $\left(p<0.001 ; R^{2}{ }_{A d j}=0.18\right)$. Thus, when symptoms decreased in one session, reflection 2 IMs increased in the next session. However, this model explained considerably less variance than the previous model ( $54 \%$ in the former, $18 \%$ in the later). Symptoms also emerged as a significant predictor of Reflection 1 IMs $\left(p<0.001 ; R_{A d j}^{2}=0.05\right)$; however, in this case, the association was positive. Therefore, when symptoms increased in one session, reflection $1 \mathrm{IMs}$ also increased in the next session. Interestingly, the variance explained by the model is very low $(5 \%)$.

In sum, hypothesis 1 (IMs were reliably identified), 2 (IMs were better predictors of symptom improvement, than the reverse) and 3 (only high-level IMs were predictive of symptom improvement) were supported. Two results were different than we expected: the diversity of IMs is reduced, and as such we only tested reflection 1 and $2 \mathrm{IMs}$, and when symptoms increase, there is also an increase in the production of reflection $1 \mathrm{IMs}$ in the following session.

Table 4. HLM with the proportion of reflection IMs as predictors of symptomatology (OQ-10.2 scores)

\begin{tabular}{lcccc}
\hline Models and fixed effects & Coefficient & $S E$ & $t$ & $p$ \\
\hline Reflection 1 IMs predicting OQ-10.2 model & & \\
Intercept ( $\beta$ 00) & 17.92 & 2.64 & 6.78 & $<0.001$ \\
Reflection 1 IMs ( $\beta$ 01) & -0.003 & 0.11 & 0.03 & 0.98 \\
Reflection 2 IMs predicting OQ-10.2 model & & \\
Intercept ( 3 00) & 19.37 & 2.29 & 8.46 & $<0.001$ \\
Reflection 2 IMs ( $\beta$ 01) & -0.28 & .08 & -3.51 & $<0.001$ \\
\hline
\end{tabular}

HLM = hierarchical linear modelling; IMs, innovative moments. 
Table 5. HLM with symptomatology (OQ 10.2 scores) as predictors of the proportion of reflection IMs

\begin{tabular}{|c|c|c|c|c|}
\hline Models and fixed effects & Coefficient & $S E$ & Z & $P$ \\
\hline \multicolumn{5}{|c|}{ OQ-10.2 predicting Reflection 1 IMs model } \\
\hline $\begin{array}{l}\text { Intercept }\left(\begin{array}{ll}\beta & 00\end{array}\right) \\
\left.\text { OQ-10.2 ( } \begin{array}{ll}\beta & 01\end{array}\right)\end{array}$ & $\begin{array}{r}-2.77 \\
0.01\end{array}$ & $\begin{array}{l}0.17 \\
0.001\end{array}$ & $\begin{array}{r}-16.15 \\
11.19\end{array}$ & $\begin{array}{l}<0.001 \\
<0.001\end{array}$ \\
\hline $\begin{array}{l}\text { OQ-10.2 predicting Reflec } \\
\text { Intercept }\left(\begin{array}{ll}\beta & 00) \\
\text { OQ-10.2 }\left(\begin{array}{ll}\beta & 01\end{array}\right)\end{array}\right.\end{array}$ & $\begin{array}{l}\text { ion } 2 \text { IMs ms } \\
-1.75 \\
-0.07\end{array}$ & $\begin{array}{l}\text { del } \\
0.27 \\
0.001\end{array}$ & $\begin{array}{r}-6.48 \\
-62.62\end{array}$ & $\begin{array}{l}<0.001 \\
<0.001\end{array}$ \\
\hline
\end{tabular}

HLM = hierarchical linear modelling; IMs, innovative moments

\section{DISCUSSION}

Data clearly show that IMs also emerge in CBT and their identification revealed a high agreement between coders. Thus, regarding the occurrence of IMs, the results of this study are comparable to those found in previous samples of NT (Gonçalves et al., 2016; Matos et al., 2009), CCT (Gonçalves et al., 2012) and EFT (Mendes et al., 2010). This finding provides further empirical support for the hypothesis that IMs represent a transtheoretical phenomenon occurring across a wide variety of psychotherapies.

To study the relationship between IMs and symptoms we replicated the analysis performed by Gonçalves et al. (2016) with NT. We tested two sets of models to evaluate the temporal relationship between these variables. That is, we first tested whether reflection 1 and 2 IMs were predictors of symptoms in the next session, and then, we tested the reverse scenario with symptoms as predictors of reflection 1 and $2 \mathrm{IMs}$ in the next session. The findings revealed that reflection 2 IMs predicted symptoms reduction in the next session, and symptom improvement in one session predicted an increase in reflection 2 IMs in the next one, suggesting a bidirectional influence. Thus, when a client spends more time elaborating on reflection $2 \mathrm{IMs}$ in one session, there is a decrease in symptoms in the next session, and when symptoms decrease in one session, there is also an increase in reflection 2 IMs in the next one. However, the variance explained for each model is quite different suggesting that IMs more strongly influence symptoms change in the following session, than the reverse (symptoms influencing IMs in the next session). Despite the fact that this is an exploratory study, it is interesting that in a model of therapy in which the therapist is apparently unaware of IMs (contrarily to what occurs with NT), IMs still predict symptom improvement. This suggests that not only IMs occur frequently in other models of therapy, as previous research has shown (Gonçalves et al., 2012; Gonçalves et al., 2016; Matos et al., 2009; Mendes et al., 2010), as IMs may have a causal role in producing change. Thus, these results reinforce the hypothesis that IMs are not just byproducts of the change process but may be a necessary element for the therapeutic change to occur.
In CBT, reflection $2 \mathrm{IMs}$ may occur as alternative thoughts emerge, which is congruent with studies revealing an association between a change in beliefs and a subsequent change in depressive symptoms (Hollon \& Beck, 2013). However, our data suggest that two particular changes in cognitions are relevant, as they occur in reflection 2 IMs (see the definition of IMs, Table 1): descriptions of positive contrasts in the self and descriptions of change processes. We hypothesize that when CBT clients describe contrasts in their self, they are narrating changes in cognitions as meaningful self-transformations (e.g., 'I'm more assertive now, I'm not afraid anymore of what others may think if I speak up'). Reflection 2 IMs also emerge when the clients describe processes of change, as in the following example: 'After our sessions, I go home and remember what we have talked and that helps me to organize my ideas. That allows me to contain the negative thoughts and focus more on the things that I have to do'. This last example illustrates what Gonçalves and Ribeiro (2012) have referred to as a position of authorship, meaning that change is not just something that occurred; it is something that the client has proactively carried out. While these two changes in cognitions (contrasts and processes of change) are related to symptom improvement, it is interesting that reflection $1 \mathrm{IMs}$ did not predict a change in symptoms in the following session (as occurred with reflection 2 IMs). Reflection 1 IMs in CBT are most likely associated with the identification of negative thoughts, e.g., 'I'm always afraid that others will think I'm stupid and that has affected me a lot.' Thus, our findings suggest that if clients cannot follow the movement from the identification of negative thoughts (reflection $1 \mathrm{IMs}$ ) to the elaboration of contrasts and the description of the change processes (reflection $2 \mathrm{IMs}$ ), then a reduction in symptoms is not likely to occur.

The relationship between increase in symptoms and production of reflection $1 \mathrm{IMs}$ is difficult to interpret. Perhaps, when there was an increase in symptoms the therapist made an effort to identify alternative thoughts that may counter the deterioration, but was unable to progress from reflection 1 IMs to reflection 2 IMs. Obviously, this result is unexpected and should be the target of future studies.

We should be very cautious in drawing clinical implications from such an exploratory study, but these results might suggest that therapists should work to help clients to gradually elaborate and accommodate alternative thoughts (DeRubeis, Webb, Tang, \& Beck, 2010), allowing a different perspective of the self to be constructed during psychotherapy (i.e., reflection 2 IMs to emerge). This could be performed in CBT if the therapist follows the change in cognitions (most likely present in reflection 1 IMs) with questions centred on contrasts ('How is this different from what occurred before?') and processes ('How did you managed to achieve this change in the way you 
think?'), thus eliciting reflection 2 IMs. Moreover, the low proportion of Reflection 2 (vs. the high proportion of Reflection 1) in later stages of therapy may be operationalized as 'red flags' for the therapist to acknowledge the client's stuckness and adapt his or her intervention efforts.

One interesting finding, which clearly needs more research, is the low variability of IMs' types present. In this study, only reflection 1 and reflection 2 were analysed in the HLM models given their higher proportion. At this stage of our research, and given the limitations of this study (see below), it is difficult to speculate why this occurred. Based on previous findings, we would expect a higher proportion of reconceptualization IMs in successful cases. Although Protest 1 and 2 also had a very low proportion in this sample this was not totally unexpected as the same occurred with other samples (e.g. constructivist therapy of grief [Alves, et al., 2014], CCT, [Gonçalves et al., 2012]). In fact, taking into consideration previous research, the result that proves harder to accommodate is the scarcity of reconceptualization IMs in this sample as this type of IM has been consistently found to be the most significant one in successful psychotherapy (Gonçalves et al., 2016; Matos et al., 2009; Mendes et al., 2010). In this sample, reflection 2 IMs, instead of reconceptualization IMs, were found to be the type most closely associated with symptomatic recovery. Until now, reconceptualization IMs were found to be fundamental in the successful outcomes of different therapeutic models, but all of these models were within the constructivist paradigm (NT, constructivist therapy, EFT and CCT). This is the first time that reconceptualization IMs did not significantly differentiate recovered from unchanged cases; however, this is also the first time that a therapeutic model outside of the constructivist paradigm (CBT) was studied. Thus, future studies with CBT could clarify whether reconceptualization IMs do not play such a relevant role in change within CBT, or whether this finding was specific to this particular small sample. In a recent CBT case study (Gonçalves, Batista \& Freitas, in press), it was found that the absence of reconceptualization was not only associated with a reduced narrative change evaluated with the Core Conflictual Relationship Theme method (CCRT, Luborsky, 1998), as the case had a relapse in the follow-up. This may suggest that the absence of reconceptualization IMs could be associated with a more superficial change with less intrapersonal change. This is probably more related with client's variables (e.g., stages of change; Norcross, Krebs, \& Prochaska, 2011), than to the particular model of therapy. Of course, this suggestion needs to be further studied in the future with extended samples.

We hypothesize that in the recovered cases of this sample, the construction of alternative thoughts and beliefs that enable a new framework of meaning to emerge was captured by reflection 2 IMs. In fact, reconceptualization IMs involve the articulation of the two components that can also be depicted by reflection 2 IMs (contrast between past and present self and elaboration of the change process). When these two components emerge together in clients' conversation that excerpt is coded as a reconceptualization IM; however, when they emerge separately, each component is coded as a reflection 2 IM (see Gonçalves et al., 2011 and Gonçalves et al. (in press) for further specifications on the coding procedure). In previous studies, we hypothesized that reflection 2 IMs are a developmental precursor of reconceptualization IMs as therapy progresses (Alves et al., 2013; Gonçalves et al., 2016; Mendes et al., 2011). Thus, if these results are replicated, and reconceptualization IMs do not emerge in new samples, this may mean that the construction of change may be facilitated through different types of IMs. Nevertheless, we speculate that the transformation of the maladaptive framework of meaning in psychotherapy always involves high level IMs (reflection 2, protest 2, reconceptualization, or action 2 IMs). This claim, as well the idea that the absence of reconceptualization IMs could be associated with more superficial change, should be investigated in future studies.

\section{Limitations}

This is an exploratory study, and as such, it contains several fragilities highlighted in this section. Moreover, the less diverse profile of IMs in this sample, when compared with previous samples, creates the need to conduct other studies with CBT. In this study, we have only one therapist and a very small number of clients. Thus, these results may be more related to therapist idiosyncrasies, than to specificities of the therapeutic model. Moreover, the small sample size also precludes any generalizability of findings. The use of self-report measures (OQ-45) is also a limitation, which is balanced by the use of observational measures, like the IMCS. Even though this research's contribution to our knowledge of the therapeutic processes is very limited, we suggest that this study has two important findings. First, IMs occur and may be reliably coded in CBT, and second, the pattern of reflection 2 predicting symptom improvement is congruent with a previous study (Gonçalves et al., 2016) in which IMs emerged as significant predictors of symptom change. Despite the limitations, we believe that this study represents an effort in the right direction.

\section{REFERENCES}

Alves, D., Fernández-Navarro, P., Baptista, J., Sousa, I., Ribeiro, E., \& Gonçalves, M. M. (2014). Innovative moments in grief therapy: The meaning reconstruction approach and the processes of self-narrative transformation. Psychotherapy Research, 24, 25-41. 
Alves, D., Mendes, I., Gonçalves, M. M., \& Neymeier, R. (2012). Innovative moments in grief therapy: Reconstructing meaning following perinatal death. Death Studies, 36, 795-818.

American Psychiatric Association (2000). DSM-IV-TR: Diagnostic and statistical manual of mental disorders(4th Revised edition.). Washington, DC: American Psychiatric Press Inc.

Beck, A. T., Rush, A. J., Shaw, B. E., \& Emery, G. (1979). Cognitive therapy of depression. New York: Guilford Press.

Beck, A. T., Steer, R. A., \& Brown, G. K. (1996). Manual for the Beck Depression Inventory-II (BDI-II). San Antonio, TX: Psychological Corporation.

Boritz, T., Bryntwick, E., Angus, L., Greenberg, L. S., \& Constantino, M. J. (2013). Narrative and emotion process in psychotherapy: An empirical test of the narrative-emotion process coding system (NEPCS). Psychotherapy Research. Advance online publication. DOI:10.1080/10503307.2013.851426.

Brinegar, M. G., Salvi, L. M., Stiles, W. B., \& Greenberg, L. S. (2006). Building a meaning bridge: Therapeutic progress from problem formulation to understanding. Journal of Counseling Psychology, 53, 165-180.

Campos, R. C., \& Gonçalves, B. (2011). The Portuguese version of the Beck Depression Inventory-II (BDI-II). European Journal of Psychological Assessment, 27, 258-264.

Coelho, R., Martins, A., \& Barros, H. (2002). Clinical profiles relating gender and depressive symptoms among adolescents ascertained by the Beck Depression Inventory II. European Psychiatry, 17, 222-226.

Crits-Christoph, P., Gibbons, M. B., \& Mukherjee, D. (2013). Psychotherapy process-outcome research. In Lambert, M. J. (Ed.), Bergin and Garfield's handbook of psychotherapy and behavior change (6th ed. pp. 298-340). Mahwah, NJ: Wiley.

de Jong, K., Nugter, M. A., Polak, M. G., Wagenborg, J. E. A., Spinhoven, P., \& Heiser, W. J. (2007). The Outcome Questionnaire (OQ-45) in a Dutch population: A cross-cultural validation. Clinical Psychology \& Psychotherapy, 14, 288-301. DOI:10.1002/cpp.529.

DeRubeis, R. J., Webb, C. A., Tang, T. Z., \& Beck, A. T. (2010). Cognitive therapy. In Dobson, K. S. (Ed.), Handbook of cognitive-behavioral therapies (3rd ed. pp. 277-316). New York, NY: Guilford Press.

First, M. B., Gibbon, M., Spitzer, R. L., Williams, J., \& Benjamin, L. (1997). Structured Clinical Interview for DSM-IV Axis II personality disorders: SCID-II. Washington, DC: American Psychiatric Press.

First, M. B., Spitzer, R., Gibbons, M., \& Williams, J. (2002). Structured clinical

Goates-Jones, M., \& Hill, C. E. (2008). Treatment preference, treatment-preference match, and psychotherapist credibility: Influence of session outcome and preference shift. Psychotherapy: Theory, Research, Practice, Training, 45, 61.

Gonçalves, M. M., Baptista, J., \& Freitas, S. (in press). Narrative and clinical change in CBT: A comparison of two recovered cases. Journal of Constructivist Psychology.

Gonçalves, M., Bento, T., Lopes, R., \& Salgado, J. (2009). Manual da Escala de Adesão e Competência [Adherence and Competence Scale for Narrative and Cognitive-Behavioral Therapy]. Braga, Portugal.

Gonçalves, M. M., Matos, M., \& Santos, A. (2009). Narrative therapy and the nature of "innovative moments" in the construction of change. Journal of Constructivist Psychology, 22, 1-23.

Gonçalves, M. M., Mendes, I., Cruz, G., Ribeiro, A. P., Sousa, I., Angus, L. E., \& Greenberg, L. S. (2012). Innovative moments and change in client-centered therapy. Psychotherapy Research, 22, 389-401.
Gonçalves, M. M., Mendes, I., Ribeiro, A., Angus, L., \& Greenberg, L. (2010). Innovative moments and change in emotion-focused therapy: The case of Lisa. Journal of Constructivist Psychology, 23, 267-294.

Gonçalves, M. M., Ribeiro, A. P., Mendes, I., Alves, D., Silva, J., Rosa, C., Batista, J., Navarro-Fernandez, P., \& Braga, C. (in press). Innovative moments, ambivalence and ambivalence resolution: Coding systems and main findings. Psychotherapy Research.

Gonçalves, M. M., Ribeiro, A. P., Silva, J., Mendes, I., \& Sousa, I. (2016). Narrative innovations predict symptom improvement: Studying innovative moments in narrative therapy of depression. Psychotherapy Research, 26, 425-435. DOI:10.1080/ 10503307.2015.1035355.

Gonçalves, M. M., \& Ribeiro, A. P. (2012). Therapeutic change, innovative moments and the reconceptualization of the self: A dialogical account. International Journal of Dialogical Science, 1, 81-98.

Gonçalves, M. M., Ribeiro, P. A., Mendes, I., Matos, M., \& Santos, A. (2011). Tracking novelties in psychotherapy process research: The innovative moments coding system. Psychotherapy Research, 21, 497-509.

Graffar, M. (1956). Une méthode de classification sociale d'echantillons de population. Courrier, 6, 455-59.

Hill, C. E., \& Lambert, M. J. (2004). Methodological issues in studying psychotherapy processes and outcomes. In Lambert, M. J. (Ed.), Handbook of psychotherapy and behavior change (5th ed. pp. 84-135). New York: Wiley.

Hollon, S. D., \& Beck, A. T. (2013). Cognitive and cognitivebehavioral therapies. In Lambert, M. J. (Ed.), Bergin and Garfield's handbook of psychotherapy and behavior change (6th ed. pp. 393-432). Mahwah, NJ: Wiley interview for DSM-IV-TR Axis I Disorders, research version, non-patient edition (SCIDI/NP). New York: Biometrics Research, New York State Psychiatric Institute.

Jacobson, N. S., \& Truax, P. (1991). Clinical significance: A statistical approach to defining meaningful change in psychotherapy research. Journal of Consulting and Clinical Psychology, 59, 12-19. DOI:10.1037/0022-006X.59.1.1.

Kanter, J. W., Busch, A. M., \& Rusch, L. C. (2009). Behavioral activation. In Dryden, W. (Ed.), The CBT distinctive features series. London: Routledge.

Lambert, M. J., Burlingame, G. M., Umphress, V., Hansen, N. B., Vermeersch, D. A., Clouse, G. C., \& Yanchar, S. C. (1996). The reliability and validity of the Outcome Questionnaire. Clinical Psychology \& Psychotherapy, 3, 249-258. DOI:10.1002/(SICI) 1099-0879(199612).

Lambert, M. J., Finch, A. M., Okiishi, J., \& Burlingame, G. M. (2005). Administration and scoring manual for the OQ-30.2. American Professional Credentialing services, LLC.

Leahy, R. L., \& Holland, S. J. (2000). Treatment plans and interventions for depression and anxiety disorders. New York: Guilford.

Lopes, R., Gonçalves, M. M., Machado, P. P. P., Sinai, D., Bento, T., \& Salgado, J. (2014). Narrative therapy vs. Cognitivebehavioral therapy for moderate depression: Empirical evidence from a controlled clinical trial. Psychotherapy Research, 24, 662-674.

Lopes, R. T., Gonçalves, M. M., Fassnach, D., Machado, P. P., \& Sousa, I. (2015). Time to improve and recover from depressive symptoms and interpersonal problems in a clinical trial. Clinical Psychology and Psychotherapy, 22, 97-105.

Luborsky, L. (1998). The early life of the idea for the core conflictual relationship theme method. In Luborsky, L., \& CritsChristoph, P. (Ed.), Understanding transference: The core 
conflictual relationship theme method (2nd ed. pp. 151-163). Washington DC: American Psychological Association.

Machado, P. P., \& Fassnacht, D. (2014). The Portuguese version of the Outcome Questionnaire (OQ-45): Normative data, reliability, and clinical significance cut-offs scores. Psychology and Psychotherapy: Theory, Research and Practice. DOI:10.1111/papt.12048.

Matos, M., Santos, A., Gonçalves, M. M., \& Martins, C. (2009). Innovative moments and change in narrative therapy. Psychotherapy Research, 19, 68-80.

Mendes, I., Ribeiro, A. P., Angus, L., Greenberg, L. S., \& Gonçalves, M. M. (2010). Narrative change in emotionfocused therapy: How is change constructed through the lens of the Innovative Moments Coding System? Psychotherapy Research, 20, 692-701.

Mendes, I., Ribeiro, A. P., Angus, L., Greenberg, L., Sousa, I., \& Gonçalves, M. M. (2011). Narrative change in emotionfocused psychotherapy: A study on the evolution of reflection and protest innovative moments. Psychotherapy Research, 21, 304-315.
Norcross, J. C., Krebs, P. M., \& Prochaska, J. O. (2011). Stages of change. In Norcross, J. C. (Ed.), Psychotherapy relationships that work: Evidence-based responsiveness (2nd ed. pp. 279-300). New York: Oxford.

Osborne, J. W. (2000). Advantages of hierarchical linear modeling. Practical Assessment, Research E Evaluation, 7(1). Retrieved October 14, 2015 from http:/ /PAREonline.net/getvn.asp? $=7 \& n=1$

$\mathrm{R}$ Development Core Team (2013). The R foundation for statistical computing. Retrieved from www.Rproject.org.

Seggar, L. B., Lambert, M. J., \& Hansen, N. B. (2002). Assessing clinical significance: Application to the Beck Depression Inventory. Behavior Therapy, 33, 253-269. DOI:10.1016/S0005-7894 (02)80028-4.

Steer, R. A., Brown, G. K., Beck, A. T., \& Sanderson, W. C. (2001). Mean Beck Depression Inventory-II scores by severity of major depressive episode. Psychological Reports, 88, 1075-1076. DOI:10.2466/pr0.2001.88.3c.1075.

White, M., \& Epston, D. (1990). Narrative means to therapeutic ends. New York: Norton. 